\title{
Investigação do processamento da compreensão linguística por meio de um software de captura
}

\author{
Investigation of linguistic comprehension processing by capture software
}

\author{
Vera Wannmacher Pereira \\ Jonas Rodrigues Saraiva
}

Pontifícia Universidade Católica do Rio Grande do Sul - PUCRS - Porto Alegre - Rio Grande do Sul - Brasil

\begin{abstract}
Resumo:A finalidade deste artigo é mostrar uma investigação semântica cognitiva sobre a ideia de expectativa, definida como um construto mental que organiza o pensamento e serve de motivação específica para a comunicação. A expectativa pode ser gerada na mente do falante e do ouvinte. Pode ser verbalizada ou não. Com relação a seu conteúdo, nem sempre se realiza, podendo trazer frustrações no processo comunicativo. Este estudo da expectativa sugere que é útil constituir uma teoria para explicar aspectos que outras abordagens deixam de lado. A metodologia usada privilegiou o levantamento de dados relacionados com o fenômeno estudado e os procedimentos explicativos dentro de uma abordagem cognitiva. Os enunciados são diretamente dependentes de como a mente funciona. Assim, ao estudar a fala, podemos ter acesso à mente das pessoas. A ideia de expectativa pode ser interpretada sob diferentes rótulos da semântica tradicional. Porém, há peculiaridades que só podem ser explicadas através de uma abordagem cognitiva da linguagem. Encontramos palavras e expressões nas línguas que revelam a presença de uma expectativa, como "eu acho que...", "houve uma expectativa a respeito de..." Esperase que toda resposta leve em conta alguma expectativa. Esta investigação trouxe evidências de que a expectativa mental é essencial ao ato de comunicação. Portanto, o estudo da expectativa nas línguas e na mente das pessoas é importante para as pesquisas em semântica cognitiva.
\end{abstract}

Palavras-chave:Compreensão linguística. Estratégias de leitura. Tecnologia. Software de captura.

Abstract: Among many computer tools that are provided for research development, especially with regard to language, the capture software technologies are important for the study of cognitive processes while performing activities using the computer as an electronic support. This article presents one - the capture software Snaglt - which records videos of the user's movements with the mouse, during the linguistic comprehension process, enabling analysis and reflections on the user's journey and thereby their cognitive processing. Two psycholinguistic studies developed at the Reference Center for Language Development - CELIN/ FALE/ PUCRS - used this capture software in order to examine the linguistic comprehension strategies applied by the subjects. These studies are presented for demonstration and explanation.

Keywords: Linguistic comprehension. Reading strategies.Technology. Capture software. 


\section{Introdução}

A tendência atual de maximização das interconexões e da acessibilidade ao conhecimento tem se valido dos mais diferentes suportes e das mais diversas ferramentas tecnológicas para o alcance de objetivos e metas em novos desafios propostos.

As pesquisas científicas têm se utilizado metodologicamente de diferentes tecnologias, desde os suportes mais complexos e dispendiosos ao mais simples software livre, a fim de abranger campos, objetos e situações de estudo até então não explorados ou de difícil exploração.

Não só na área da linguagem, o computador se configura como suporte tecnológico indispensável para qualquer iniciativa, seja científica ou não, somando-se a ele, atualmente, as mais diversas modalidades e potencialidades, como notebooks, tablets, e-readers(...) e até smartphones. Os softwares e aplicativos que podem integrá-lo(s) constituem-se em ferramentas que abrem caminhos por vezes muito difíceis de acesso.

No caso dos estudos aqui relatados, o computador é o suporte eletrônico e o software de captura Snaglt é a ferramenta que permite acompanhar, via gravação em vídeo da tela, os passos do usuário e, por consequência, seu percurso cognitivo.

É esse o ponto chave para as pesquisas desenvolvidas no Centro de Referência para o Desenvolvimento da Linguagem - CELIN da FALE/PUCRS, tendo em vista apoiarem-se na Psicolinguística, que é a área da Linguística em interface com a Psicologia que busca estudar os processos envolvidos nas relações entre mente/cérebro e linguagem. Mais especificamente, as propostas de pesquisa relatadas focalizam o uso das estratégias de leitura, na medida em que seu desfilamento contribui para 0 avanço dos conhecimentos psicolinguísticos sobre 0 processamento da compreensão nos distintos públicos - estudantes da educação básica, graduandos, professores...

Com o propósito de demonstração da contribuição do Snaglt para estudos psicolinguísticos de compreensão da linguagem, são apresentados sucessivamente 0 objeto teórico de estudo (compreensão, processamento e estratégias), o software, duas investigações que o utilizaram e as considerações finais.

\section{0 objeto teórico de estudo}

Os pesquisadores em Psicolinguística, para o entendimento do processamento da linguagem, têm desempenhado muitas ações de pesquisa em busca do conhecimento dos processos cerebrais/mentais da linguagem e da língua. A maioria dessas iniciativas científicas se dá de forma aplicada e, no caso do CELIN, de forma envolvida com o ensino. Isso será demonstrado nos tópicos posteriores que tratarem dos estudos já desenvolvidos. Cabe registrar que o estudo do processamento da linguagem pode contribuir para o entendimento dos processos de aprendizagem e também para o crescimento desses processos de forma a melhorar os níveis de leitura e compreensão dos alunos brasileiros, que, atualmente, encontram-se baixos.

Para estudar o processamento cognitivo, a visão dos estudos que serão apresentados é a de que a compreensão é o encontro das informações do texto com as informações prévias do indivíduo. Nessa visão, esse encontro se dá em dois processos cognitivos denominados bottom-up e top-down.

O processamento bottom-upse caracteriza por um percurso ascendente, ou seja, da informação para a cognição. Reflete o momento em que o leitor, por exemplo, realiza a decodificação e a compreensão das palavras e da estrutura de um texto. É um processo de composição, uma vez que as partes gradativamente vão formando o todo.

O processo top-down - Goodman (1991) e Smith (1999) - caracteriza-se como um movimento não linear que faz uso de informações que vêm dos conhecimentos do próprio usuário, tendo sido requisitadas pelas informações adquiridas pelo processamento bottom-up. Desse modo, dá-se o percurso do indivíduo para o texto. 
O modelo de Goodman baseia-se na concepção antecipatória, segundo a qual são utilizadas, simultaneamente, as informações grafofônicas, sintáticas (padrões sentenciais, marcadores desses padrões e regras transformacionais) e semânticas (vocabulário, conceitos e experiências do leitor).

Ainda segundo Goodman (1991), o processo cognitivo de compreensão se altera, a partir de algumas variáveis: objetivo da ação de compreender, conhecimento prévio do conteúdo e da língua, condições de produção do texto, tipo/gênero de texto e estilo cognitivo de quem está disposto a compreender o texto. Tais variáveis determinam o processo de compreensão - ascendente ou descendente - e a escolha das estratégias cognitivas e metacognitivas (SMITH, 1999, 2003).

Segundo a visão psicolinguística adotada nas pesquisas realizadas no CELIN, há um processo interativo entre os dois movimentos e, mais especificamente para seu estudo, uma inter-relação deles com as estratégias de leitura - explicitadas mais adiante. Assim, o processo de compreensão é construído a partir das formas materiais (gráficas ou fônicas) que interagem com todas as conexões geradas - no momento de compreensão ou previamente - no cérebro. Assim, os dois movimentos ocorrem de modo que o processamento ascendente indica a sensibilidade do ouvinte para a informação nova ou que não corresponde a uma hipótese formulada e o processamento descendente contribui para a solução de ambiguidades e para a seleção entre possibilidades de entendimento (KLEIMAN, 1989; LEFFA, 1996).

Nesse sentido, o sucesso da compreensão está na escolha do processo e das estratégias mais eficientes para dar conta dessa situação, em que variáveis se inter-relacionam e influenciam as escolhas do sujeito.

Como já referido anteriormente, as estratégias de compreensão podem ser cognitivas e metacognitivas. As cognitivas caracterizam-se pela ocorrência inconsciente por parte do usuário, enquanto as metacognitivas caracterizam-se pela consciência, ou seja, pela intenção de monitoramento do próprio processo.

Estudos realizados sobre esse tópico apresentam diferentes categorizações de estratégias. Neste estudo e nos projetos que serão mencionados, verificaram-se como mais recorrentes as seguintes: scanning, que secaracteriza pelo direcionamento na busca por alguma informação específica no material linguístico em questão (escrito, auditivo...); compreensão detalhada, que se caracteriza por uma abordagem atenta, minuciosa, linear do material linguístico, buscando a compreensão de todas as informações disponíveis; automonitoramento, que consiste na observação contínua do usuário com relação aos processos que está realizando durante a depreensão das informações; autoavaliação, que, subsequentemente aoautomonitoramento, consiste numa forma de verificar a produtividade da compreensão; autocorreção, que, subsequentemente às estratégias de automonitoramente e autoavaliação, constitui-se numa forma de correção das "rotas" de compreensão, com base na avaliação da produtividade dos raciocínios já realizados; predição, que se caracteriza por antecipações das informações do material linguístico, advindas de pistas linguísticas em associação a conhecimentos prévios; inferência, que consiste na realização de percursos cognitivos propiciados por pistas do material linguístico, permitindo conclusões dedutivas a partir de associação de proposições.

A partir do estudo dessas estratégias, e com o uso da tecnologia de gravação dos movimentos dos usuários no computador, é possível identificar uma hipótese do percurso cognitivo desses usuários durante o processamento da linguagem. É um percurso de observação e análise, possibilitado, no caso dos estudos desenvolvidos no CELIN, pela ferramenta Snaglt. Sendo assim, cabe uma apresentação mais detalhada desse software, o que é feito no tópico seguinte. 


\section{0 software de captura}

Um software de captura é um aplicativo que permite a digitalização de dados físicos. Os softwares utilizados por mecanismos de escaneamento (scanners, leitores de códigos de barra...) são exemplos disso.

Esses softwares também permitem a captura estática ou dinâmica da tela do computador, dependendo do objetivo desse processo por parte do usuário, no caso, o pesquisador. Em qualquer computador, o uso da tecla Print Screené um comando disponível de gravação estática da imagem que aparece na tela no momento do comando. É um meio simples e livremente disponível para a gravação de um documento, de uma página de respostas, de um texto escrito, etc.

Porém, se o objetivo, como é o caso das pesquisas psicolinguísticas, é a observação do processo, pode então ser utilizada uma ferramenta que capture a imagem dinâmica da tela, ou seja, um tipo de gravação de vídeo de toda a movimentação utilizando a máquina, por meio das imagens da tela.

Existem vários softwares que permitem tal procedimento - Camtasia Studio; Bandicam; TakeScreenLitlle; ScreenHunter; ScreenSnag; CaptureWiz; SnapCrab; Fraps e Hyper can. Um deles é o Snaglt, software utilizado nos projetos do Centro.

Desenvolvido pela TechSmith Corporation, seu nome, em tradução livre, pode estar relacionado a "puxar" ou "prender" a imagem da tela. As funções que oferece, apresentadas em uma interface bastante intuitiva, são: opções de definição da área de captura, conforme mostra a Figura 1, e opções de tipo de captura - texto; vídeo; imagem - e qualidade de saída, conforme mostra a Figura 2.

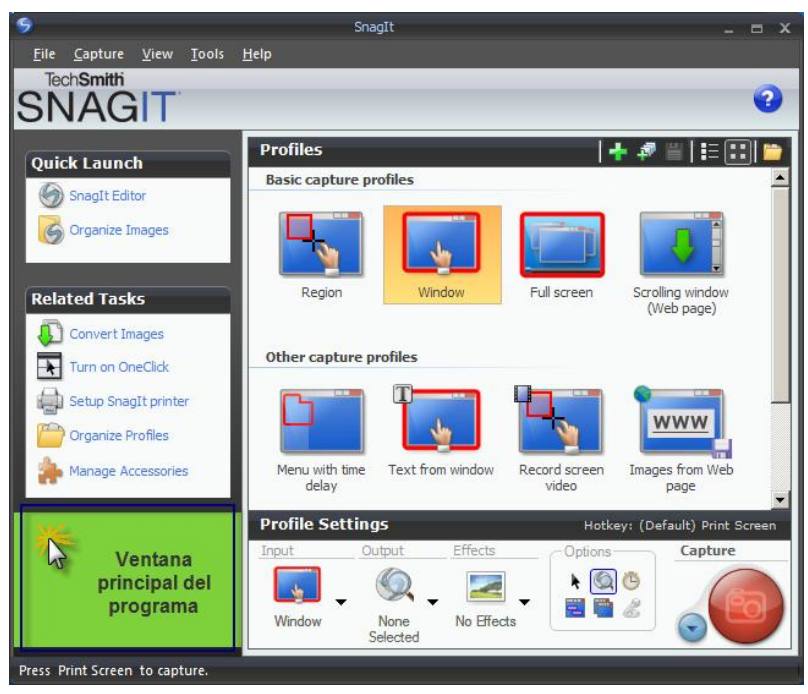

Figura 1: Opções de área de captura.

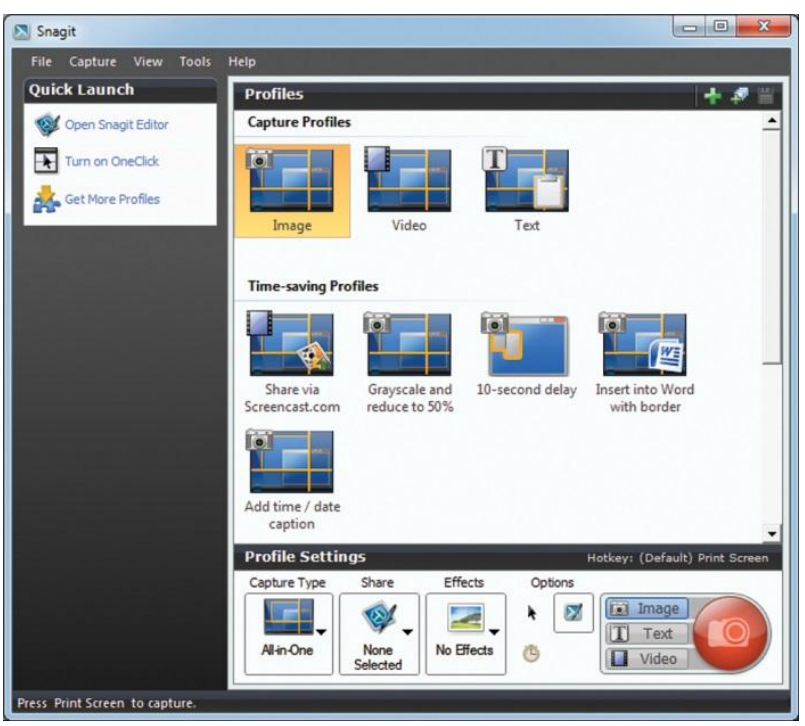

Figura 2: Formatos de captura disponíveis.

Uma das funcionalidades do Snaglt, assim como de outros programas de captura de tela é a produção de vídeos demonstrativos, ou, como são conhecidos, principalmente no Youtube, videoaulas. Para a pesquisa, a utilidade do software se dá no momento da coleta de dados no computador, tendo em vista que ele possibilita, como dito, a gravação das ações do sujeito durante a aplicação de um instrumento de pesquisa, por exemplo. Assim, podese acompanhar a movimentação do cursor do mouse e da tela em si (movimentação da barra de rolagem, acesso a links, utilização de botões e comandos...) durante a compreensão de um texto, a realização da atividade de um objeto de aprendizagem, o preenchimento de um formulário, a navegação em um site, etc. 
É possível verificar, por exemplo, a utilização das estratégias durante a compreensão de um texto. Hipotetizando uma situação de compreensão virtual, se o leitor utilizar o cursor para acompanhar os movimentos oculares durante o procedimento de compreensão (e isso pode ser solicitado ao sujeito), o pesquisador terá acesso às características desse processo. Se o sujeito avançar no acompanhamento do texto, de forma que se note que está "passando os olhos", pode estar utilizando uma estratégia de skimming ou de scanning, por exemplo. Ou ainda, se percorrer cada palavra e cada linha do texto, atentamente, pode estar realizando análise detalhada. Se precisar retomar uma parte do texto e subir rapidamente o cursor na página, ou, ainda, se utilizar a barra de rolagem, pode estar utilizando automonitoramento, seguido de autocorreção. Se dá saltos pequenos e avança no texto, pode estar utilizando predição leitora, e assim por diante.

Esses dados podem ficar mais claros na descrição dos projetos que utilizaram o recurso de captura de tela possibilitado pelo Snaglt. A seguir, são apresentados alguns desses projetos.

\section{Dois estudos com uso do software de captura}

Como dito, o CELIN desenvolve muitas pesquisas na área da Linguística, mais especificamente da Psicolinguística, e procura focalizar suas iniciativas no eixo ensino, pesquisa e extensão. Assim, os projetos são desenvolvidos em parceria com escolas públicas e privadas e contam com a participação de acadêmicos de graduação, de pós-graduação e professores.

O Centro possui a característica inerente de voltar suas investigações ao ensino, variando o público-alvo para atingir diferentes aspectos e tópicos da educação básica e superior. Além disso, a maioria dos projetos solicita algum tipo de recurso tecnológico para determinados aspectos da metodologia, em geral o computador, o Snaglt..., como é o caso dos projetos apresentados a seguir, desenvolvidos no CELIN com essas características.

\subsection{Estudo com professores: processamento da compreensão leitora de texto linear e de texto em mapa conceitual}

Uma das primeiras pesquisas desenvolvidas no Centro com o uso do Snaglt foi o Projeto Compreensão e processamento da leitura de e-book: estratégia, inferência, relevância e satisfação, desenvolvido entre 2009 e 2010. O projeto foi uma iniciativa que envolveu as Faculdades de Letras (FALE) e de Informática (FACIN) da PUCRS. Seu objetivo foi investigar o uso de um e-book, sobre ensino da leitura, por professores de Língua Portuguesa do Ensino Fundamental, no que se refere à compreensão leitora e ao processamento da leitura (estratégia de leitura, inferência, relevância e satisfação), nos formatos em Mapa Conceitual e em Texto Linear.

O projeto recebeu aporte interdisciplinar, já que uniu o referencial teórico vinculado à Psicolinguística, já mencionado, anteriormente, neste texto, o referencial da área da Pragmática - como suporte ao estudo dos aspectos inferência e relevância -, com base em Peirce (1975), Sperber e Wilson (1995), Grice (1975) e Costa (2005), o referencial teórico da Informática, baseado nos estudos de Mack e Nielsen (1994), Nielsen (1993) e o referencial da Educação, com apoio em estudos sobre Mapas Conceituais, como os de Ausubel (2000), Magliano, Graesser e Trabasso (1999), Moreira (1980), Moreira e Masini (2006) e Novak (1996).

O e-book, desenvolvido para uso no projeto, recebeu o título Leitura e Cognição: teoria e prática nos anos finais do Ensino Fundamental e está disponível para estudos no site da EDIPUCRS nos dois formatos (linear e mapa conceitual) ${ }^{1}$. É constituído de setenta e três páginas, o que representa um número bastante expressivo para uma situação de pesquisa. Por esse motivo, a equipe de pesquisa decidiu centrar sua investigação em três dos capítulos do livro - Leitura: tipos e gêneros textuais, Leitura: planos linguísticos e Leitura: compreensão,

\footnotetext{
${ }^{1}$ http://www.pucrs.br/edipucrs/leituraecognicao/e_book_2009.ht $\mathrm{ml}$;

http://www.pucrs.br/edipucrs/leituraecognicaomc/index.html.
} 
processamento e estratégias -, pois apresentavam fundamentação teórica necessária para o ensino da leitura em sala de aula e atividades práticas de leitura. Assim, a investigação era composta pela leitura desses três capítulos, antecedida pelos elementos pré-textuais (capa, folha de rosto, ficha catalográfica), pelo capítulo Abertura, pelo capítulo

Mapa de Navegação, e em seguida pela leitura do capítulo final, Fechamento.

Os instrumentos de investigação utilizados com os sujeitos (20 professores de Língua Portuguesa nos anos finais do Ensino Fundamental, $5^{\underline{a}}$ a $8^{\underline{a}}$ séries) foram um Teste de Compreensão Leitora (TCL), um Questionário de Adesão sobre o formato de e-book, eo próprio e-book, todos sob o monitoramento do software de captura. Como o foco do presente artigo é justamente a utilização desse software, será interessante abordar aspectos relacionados à compreensão e ao processamento. Por isso, não serão mencionados dados referentes aos demais instrumentos além de alguns do de compreensão e as informações adquiridas pelo Snaglt.

O Teste de Compreensão Leitora (TCL) foi desenvolvido em forma de formulário eletrônico, em linguagem $\mathrm{C}++$, no ambiente Visual Studio, composto por doze questões, estando estas distribuídas em grupos de quatro questões que contemplam os temas (a) gêneros textuais, (b) planos linguísticos e (c) compreensão, processamento e estratégias de leitura.

Levando em conta os dois formatos do e-book - Texto Linear em HTML e Mapa Conceitual -, os vinte sujeitos foram distribuídos em dois grupos. Um grupo realizou a leitura dos três capítulos no e-book emformato linear, e um segundo grupo efetuou a leitura dos capítulos no e-book em formato mapa conceitual. Durante a aplicação dos testes, em diferentes horários, os usuários eram encaminhados ao CELIN, onde eram apresentados ao bolsista monitor e recebiam uma explicação de como seria o procedimento do teste. Os sujeitos eram informados de que sua interação com o computador estaria sendo gravada com o uso do software de captura
Snaglt e de que, após a finalização do teste de compreensão, responderiam ao questionário de adesão ao formato.

Após a realização dos testes, os primeiros dados analisados foram os que se referiam ao nível de compreensão leitora do e-book alcançado pelos sujeitos, nos formatos texto linear e mapa conceitual. Os resultados demonstram que a variável formato não influenciou no nível de compreensão dos mesmos, já que não houve disparidades entre os formatos $a$ e $b$. No formato mapa conceitual, a média percentual do escore de acertos foi de 50,08\% enquanto que o formato texto linear atingiu média percentual de 49,92\%.

Com relação às características das estratégias utilizadas pelos sujeitos durante a leitura dos formatos a e b, a partir dos vídeos de captura, puderam-se obter tais dados e concluir quais estratégias foram mais utilizadas e, portanto, determinar o tipo de processamento predominante. Assim, as estratégias de leitura detalhada, com número de ocorrências de 2503 e tempo de utilização de 106.737 segundos, de autoavaliação, com número de ocorrências de 770 e tempo de utilização de 19.147 segundos, e de scanning, com número de ocorrências de 358 e tempo de utilização de 5.135 segundos, foram as mais utilizadas. As estratégias de automonitoramento e skimming foram as menos utilizadas.

Em relação às características do processamento cognitivo utilizado pelos sujeitos durante a leitura do e-book, nos dois formatos, podese afirmar, segundo a análise dos vídeos do Snaglt, que houve a predominância do processamento bottom-up - apoiado na utilização das estratégias leitura detalhada, autoavaliação e scanning.

Considerando a interferência da variável formato na satisfação, na compreensão e no processamento da leitura do e-book: em relação à satisfação, o Formato Texto Linear foi positivamente avaliado e recebeu índice de satisfação superior ao do Formato Mapa Conceitual; em relação à compreensão, não houve disparidade. O Formato Mapa Conceitual permitiu que os sujeitos obtivessem média de acertos de 50,08, enquanto a média de 
acertos dos sujeitos do Grupo Texto Linear foi de 49,92, o que corresponde a uma diferença de 0,16 pontos - apenas um acerto a mais no Formato Mapa Conceitual.

\subsection{Estudo com alunos: processamento da compreensão leitora e da compreensão auditiva}

O ProjetoE-book e A-book: compreensão, processamento e aprendizagem por alunos do Ensino Fundamental também utilizou a ferramenta Snaglt para o estudo da compreensão.

Desenvolvido entre 2011 e 2012, também integrou as áreas de Letras (FALE/PUCRS) e de Informática (FACIN/PUCRS) e teve como direção e embasamento teóricos os mesmos citados no projeto anterior referentes às áreas de Linguística (excetuando-se os conceitos ligados à Pragmática, já que não houve essa interface) e de Informática (excetuando-se os autores com estudos referentes a mapas conceituais).

Seu objetivo principal foi o de construir, com atividades virtuais de ensino de estratégias de leitura e de conteúdos linguísticos de coesão e coerência, um livro eletrônico em dois formatos: um para leitura e outro para audição e aplicá-los a um grupo de alunos de $8^{\underline{a}}$ série para verificar 0 nível de compreensão nos dois formatos e estudar as características de processamento bem como o nível de aprendizagem dos conteúdos linguísticos por parte dos sujeitos.

$\mathrm{O}(\mathrm{s})$ livro(s) utilizado(s) como principal(is) instrumento(s) do estudo está(ão) disponível(is) no site da EDIPUCRS ${ }^{2}$ e foi(foram) desenvolvido(s) a partir da seleção de textos de diferentes gêneros, sobre os quais foram elaboradas atividades com ênfase em estratégias de leitura - predição, scanning, compreensão detalhada, e um bloco com automonitoramento, autoavaliação e autocorreção - e conteúdos linguísticos de regras de coerência e

\footnotetext{
${ }^{2}$ http://ebooks.pucrs.br/edipucrs/projetos/ebookabook/ebook/V2/ index.html; http://ebooks.pucrs.br/edipucrs/projetos/ebookabook/abook/V3/a _book_2011.html.
}

coesão textual. Foram elaboradas quatro atividades para cada estratégia, totalizando dezesseis, portanto. As atividades do formato virtual de leitura foram programadas pela equipe de Informática. As atividades do formato virtual de áudio foram gravadas pela equipe de Letras.

Como sujeitos do processo, foram convidados 10 alunos de $8^{\text {a }}$ série do Ensino Fundamental de escola da comunidade, que responderam a instrumentos de avaliação da compreensão (de ambos os formatos), da aprendizagem, da satisfação, da atenção.

A coleta de dados ocorreu sob orientação dos bolsistas, individualmente, na própria escola dos sujeitos, utilizando equipamentos e ferramentas disponíveis, buscando obter informações sobre os interesses de pesquisa. Assim, a compreensão leitora foi analisada com base nos acertos das questões do instrumento; o processamento da leitura foi analisado com base no exame dos filmes obtidos pelo software de captura e com apoio nos pressupostos teóricos sobre compreensão; a aprendizagem de conteúdos linguísticos foi analisada com base nos acertos das questões do instrumento específico para esse fim, sendo os escores agrupados conforme as variáveis estabelecidas; a satisfação dos alunos com o e-book (formato e conteúdo) foi analisada mediante suas respostas ao instrumento específico para esse fim; a satisfação de professores com o trabalho proposto foi examinada com base em suas percepções sobre o ebook gerado, manifestas em instrumento específico para esse fim. 
O texto que você vai ler está completo. Porém, ao final, poderia ser acrescentada uma palavra. Isso fica claro pela estrutura da última frase. Leia o texto e descubra que palavra é essa, selecionando a alternativa correta.

Por que os escoceses usam "saia"?

Para começar, não é exatamente uma saia - ai de você se chamar o kilt de saia na frente de um escocês! No final do século 14, ele já era usado pelo povo gaélico, que vivia na Irlanda. Com a migração dos gaélicos para a região úmida e chuvosa das Highlands, no norte e no oeste da Escócia, o aparato foi adotado pelos escoceses da região. Os kilts serviam para a proteção contra a umidade e o frio típicos de lá. $\mathrm{O}$ tecido era feito de lã escovada, que impermeabilizava à água. Naquela época, a peça única era presa ao corpo, como um tipo de manto. É aí que está a origem do nome "kilt", que, na antiga língua falada na Escócia, significa o ato de "prender uma roupa no corpo". O tipo de xadrez do kilt (chamado de tartan) mudava de estampa de acordo com o cla

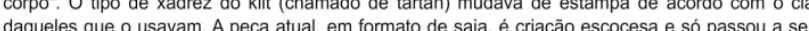
daqueles que o usavam. A peça atual, em formato de sala, e craçáo escocesa e só passou a ser usada a partir do século 18 . No século seguinte, foi adotada como símbolo de identidade naciona e hoje e vestida por cidadás escoceses e de outros palses, como Inglaterra, em ocasiōes diversas, como festas formais, eventos da moda ou pela plateia de jogos esportivos. Isso é muito
estranho para os brasileiros, mas, como visto, para outros povos, não é.

( ) Bonito

( ) Típico

( ) Estranho REF: Linardi, Fred. Revista Mundo Estranho. Ed 102. São Paulo: Agosto, 2010. (Adaptado)

\section{CONFIRMAR}

Figura 3 - Exemplo de atividade do e-book.
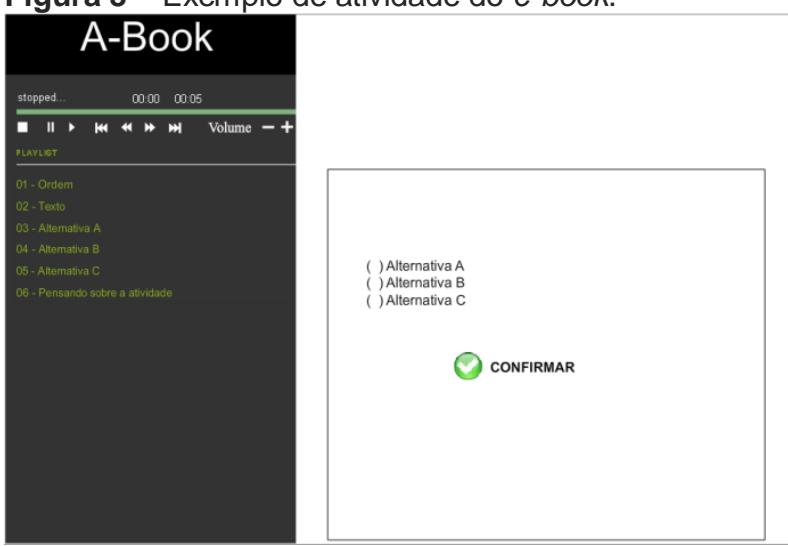

Figura 4 - Exemplo de atividade do a-book.

A análise do tipo de processamento cognitivo da compreensão predominante por parte dos sujeitos foi feita verificando-se primeiramente quais as estratégias utilizadas por eles - com base nos vídeos capturados. Pela análise, foram utilizadas as estratégias: compreensão detalhada), scanning, skimming, automonitoramento, autoavaliação e autocorreção.

Foi verificado o tempo de ocorrência de cada estratégia e a quantidade dessas ocorrências. Ao observarem-se os dados, pôde-se constatar que a estratégia de compreensão mais utilizada para os dois formatos, tanto em número de ocorrências como em tempo total de utilização, foi a de compreensão detalhada. Em segundo lugar, ficou a estratégia de automonitoramento.

A estratégia autocorreção também foi bastante empregada, sendo em maior número no ebook. Percebe-se que, ao utilizarem essa estratégia, ocorreu concomitantemente o automonitoramento e a autoavaliação.

Ao analisar os percursos das atividades do abook, é possível notar que os sujeitos iniciavam ouvindo todas as orientações dos exercícios, utilizando a estratégia compreensão detalhada, e, ao resolver as questões, colocavam o texto (áudio) novamente, automonitorando-se para responder corretamente. Caso a resposta fosse incorreta, acontecia a autocorreção. Para isso, ocorria antes uma autoavaliação da resposta, retornando ao texto (áudio) e automonitorando a compreensão.

O e-book apresentou essas mesmas características no comportamento dos sujeitos (ressalte-se que o tempo de uso de AM nos dois formatos foi muito semelhante), porém identificou-se que o tempo para resolver as questões foi menor. Supõe-se que isso tenha ocorrido pelo fato de ser mais fácil a visualização das respostas e do próprio texto, ao contrário do a-book.

Em alguns momentos foi utilizada a estratégia scanning, quando o sujeito buscava um trecho mais específico no texto. Majoritariamente isso aconteceu no a-book.

O que se pode concluir desta análise é que 0 tipo de processamento cognitivo predominante nos dois tipos de instrumentos (e-book e a-book) foi o bottom-up, pois a estratégia compreensão detalhada foi a mais utilizada em ambos os formatos, considerando que não era necessário recorrer ao conhecimento prévio para responder às questões propostas, já que as informações estavam presentes no próprio texto.

\section{Conclusões}

A metodologia de um projeto de pesquisa é o caminho que o leva a poder contribuir de alguma forma com o avanço científico, ou seja, é o que o torna relevante, o que o faz existir. Atualmente, com o máximo envolvimento da tecnologia em todas as áreas, as metodologias de pesquisa estão cada vez mais se utilizando de meios tecnológicos 
para ampliarem suas possibilidades de aplicabilidade.

Essas contribuições têm se dado nos mais diversos campos de estudo, sendo mais comuns em algumas áreas (informática, engenharias...). Porém, áreas como a Linguística têm, por meio de atividades interdisciplinares, atuado também com o uso da tecnologia para o alcance de seus objetivos científicos.

O CELIN, Centro de Referência para o Desenvolvimento da Linguagem, da Faculdade de Letras da PUCRS tem atuado nesse caminho de buscas científicas, utilizando ferramentas tecnológicas para propiciar metodologias de estudo dos processos de compreensão linguística.

Neste trabalho foram relatadas duas iniciativas de pesquisa do Centro que utilizaram a ferramenta tecnológica Snaglt, um software de captura, para gravação da tela do computador durante a aplicação de instrumentos de pesquisa. Os vídeos gerados por esse software permitiram ir ao encontro de objetivos que focalizavam a análise do processamento da compreensão por parte dos sujeitos.

No primeiro projeto relatado, cujos sujeitos eram professores de Ensino Fundamental, a utilização do Snaglt foi fundamental para o alcance dos resultados, tendo em vista a possibilidade de análise das características de uma leitura linear e de uma leitura não linear (de conceitos interligados em formato mapa conceitual). As análises dos vídeos permitiram a verificação de que a compreensão dos leitores não foi afetada no formato não linear e também de que a estratégia de leitura detalhada permanece como a mais empregada, independentemente do formato, o que sugere a utilização predominante do processamento bottom-up.

$\mathrm{Na}$ segunda pesquisa relatada, a verificação das características (semelhanças e diferenças) de um processamento de compreensão auditiva e de um processamento de compreensão leitora, por parte de um público de alunos de Ensino Fundamental, foi possibilitada também pelo uso da ferramenta, ao gravar os procedimentos dos usuários. Nesse caso, em especial, um elemento desafiador foi a análise do formato áudio, pois em princípio não apresenta elementos visuais que permitam o estudo por meio da captura de tela. Porém, as atividades foram programadas de modo que a análise da movimentação foi possível com base na movimentação do cursor do mouse e das funcionalidades disponíveis na tela (como play, pause, barra de áudio...). Assim, os dados possibilitaram as conclusões de que a estratégia de compreensão detalhada foi a mais utilizada em ambos os formatos e a de autocorreção também teve uso bastante elevado. O tipo de processamento utilizado, predominantemente também foi o bottom-up, independentemente do formato.

Esses exemplos de pesquisas endossam a possibilidade de investigação do processamento da compreensão por meio de artefatos tecnológicos, como, por exemplo, softwares de captura. Essas possibilidades não se verificam somente em estudos psicolinguísticos, mas em áreas que manifestem interesse pelo percurso virtual dos indivíduos.

Desse modo, são relevantes as propostas de investigação que possam chegar a resultados importantes para as mais diversas áreas de estudo e que, ao mesmo tempo, sejam metodologicamente inovadoras e apresentem à comunidade científica novas tecnologias para auxiliarem no desenvolvimento dos estudos. No caso dos trabalhos aqui relatados, trata-se de uma tecnologia acessível a pesquisadores de qualquer porte, que pode ser muito útil no registro das atividades do computador e propiciar o estudo dos passos dos usuários e, por conseguinte, revelar o percurso cognitivo de suas ações. 


\section{Referências}

AUSUBEL, D.P. Aquisição e retenção de conhecimentos: uma perspectiva cognitiva. Lisboa: Plátano Edições Técnicas, 2000.

COSTA, Jorge Campos da. Comunicação e inferência em linguagem natural. Letras de Hoje, Porto Alegre, n. 139 , p. 107-133, 2005.

GOODMAN. Kenneth S. Unidade na leitura - um modelo psicolinguístico transacional. Letras de Hoje, Porto Alegre, n. 86, p. 9-43, dez. 1991.

GRICE, P.; COLE, P.; MORGAN, J. (eds.). Syntax and semantics. New York: Academic Press, 1975.

KLEIMAN, Ângela. Texto e leitor: aspectos cognitivos da leitura. Campinas: Pontes, 1989.

LEFFA, Vilson J. Aspectos da leitura: uma perspectiva psicolinguística. Porto Alegre: SagraLuzzatto, 1996.

MACK, R.; NIELSEN, J. (eds.). Usability Inspection Methods. New York: John Wiley \& Sons, 1994.

MAGLIANO, J. P.; GRAESSER, A.; TRABASSO, T. Strategic processing during comprehension. Journal of Educational Psychology, vol. 9, n. 4, p. 615-629, 1999.

MOREIRA, M. A. Mapas conceituais como instrumentos para promover a diferenciação conceitual progressiva e a reconciliação integrativa. Ciência e Cultura, 32(4), p. 474-479, 1980.

MOREIRA, M.A.; MASINI, E.F.S. Aprendizagem significativa: a teoria de aprendizagem de David Ausubel. São Paulo: CentauroEditora, 2006.

NIELSEN, J. Usability Engineering. San Diego: Academic Press, 1993.

NOVAK, J.D.; GOWIN, D.B. Aprender a aprender. Lisboa: Plátano Edições Técnicas, 1996.

PEIRCE, C.S. Semiótica e filosofia. São Paulo: Cultrix, 1975.

SPERBER, D.; WILSON, D. Relevance: communication \& cognition. Mass.: Harvard University Press, 1995.

SMITH, Frank. Leitura significativa. Porto Alegre:

Artes Médicas, 1999.

SMITH, Frank. Compreendendo a leitura. Porto

Alegre: Artes Médicas, 2003. 